\title{
CAUCHY INTEGRALS AND MÖBIUS GEOMETRY OF CURVES*
}

\author{
DAVID E. BARRETT ${ }^{\dagger}$ AND MICHAEL BOLT ${ }^{\ddagger}$
}

Key words. Cauchy integral, Kerzman-Stein operator, inversive arc-length

AMS subject classifications. $30 \mathrm{C} 40$

Define a line bundle $E$ over the Riemann sphere $\widehat{\mathbb{C}}=\mathbb{C} \cup\{\infty\}$ by gluing together two copies of $\mathbb{C} \times \mathbb{C}$ using the transition mapping $(z, w) \mapsto(1 / z, i w / z)$. Since the square of this bundle is the holomorphic cotangent bundle of $\widehat{\mathbb{C}}$ it is reasonable to use the notation $f(z) \sqrt{d z}$ for sections of $E$.

If $f(z)$ is holomorphic on $R<|z|<\infty$ then $f(z) \sqrt{d z}$ extends to a holomorphic section of $E$ over $R<|z| \leq \infty$ if and only if $f(z) \rightarrow 0$ as $z \rightarrow \infty$.

A Möbius transformation $T: z \mapsto \frac{a z+b}{c z+d}$ of $\widehat{\mathbb{C}}$ lifts to (a pair of) maps $E \rightarrow E$ given by $(z, w) \mapsto\left(T z, \frac{\sqrt{a d-b c}}{c z+d} w\right)$. Note that the norm

$$
\|F\|_{\gamma}=\sqrt{\int_{\gamma}|f(z)|^{2}|d z|}
$$

of a section $F=f(z) \sqrt{d z}$ of $E$ over a curve $\gamma$ in $\widehat{\mathbb{C}}$ is Möbius-invariant.

Given a smooth Jordan curve $\gamma \subset \mathbb{C}$ and a section $F=f(z) \sqrt{d z}$ of $E$ over $\gamma$ then the Cauchy integral

$$
\frac{1}{2 \pi i} \int_{w \in \gamma} f(w) \sqrt{d w} \frac{\sqrt{d z} \sqrt{d w}}{w-z}
$$

defines a pair of holomorphic sections $\mathcal{C}_{\gamma}^{\text {in }} F$ and $\mathcal{C}_{\gamma}^{\text {out }} F$ over the two components $\gamma^{\text {in }}$ and $\gamma^{\text {out }}$ of $\widehat{\mathbb{C}} \backslash \gamma$ with the property that $F$ is the jump

$$
\text { b. v. } \text { e }_{\gamma}^{\text {in }} F-\text { b. v. } \text { C }_{\gamma}^{\text {out }} F
$$

between boundary values of $\mathcal{C}_{\gamma}^{\text {in }} F$ and $\mathcal{C}_{\gamma}^{\text {out }} F$; moreover, the operators $\mathcal{C}_{\gamma}^{\text {in }}$ and $\mathcal{C}_{\gamma}^{\text {out }}$ are completely characterized by this description. (This is a restatement of the classical Plemelj formula - see for example [Hen, §14.1].)

Since the characterization of $\mathcal{C}_{\gamma}^{\text {in }} F$ and $\mathcal{C}_{\gamma}^{\text {out }} F$ given above is Möbius-invariant it follows that the operators $\mathcal{C}_{\gamma}^{\text {in }}$ and $\mathcal{C}_{\gamma}^{\text {out }}$ are Möbius-invariant. (This can also be checked by a direct computation verifying that the Cauchy kernel $\frac{1}{2 \pi i} \frac{\sqrt{d z} \sqrt{d w}}{w-z}$ is Möbiusinvariant.)

We also consider the following Möbius-invariant operators.

\footnotetext{
${ }^{*}$ Received August 2, 2006; accepted for publication March 9, 2007.

$\dagger$ Department of Mathematics, University of Michigan, Ann Arbor, MI 48109-1043, USA (barrett@ umich.edu).

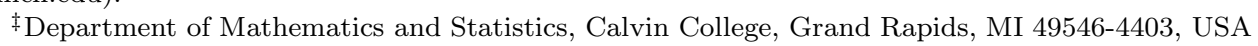
(mdb7@calvin.edu).
} 
The Cauchy operator: This is the operator $\mathcal{C}_{\gamma}$ mapping sections of $E$ over $\gamma$ to sections of $E$ over $\gamma$ given by the principal value of (2) or by the formula

$$
\mathcal{C}_{\gamma} F=\frac{1}{2}\left(\text { b. v. } \mathcal{C}_{\gamma}^{\text {in }} F+\text { b. v. } \mathcal{C}_{\gamma}^{\text {out }} F\right)
$$

[Hen, §14.1].

The Kerzman-Stein operator: This is the operator $\mathcal{A}_{\gamma}$ defined as twice the anti-self-adjoint part of $\mathcal{C}_{\gamma}$ with respect to the norm (1); if $F=f(z) \sqrt{d z}$ then $\mathcal{A}_{\gamma} F$ is given by

$$
\frac{1}{2 \pi i} \int_{w \in \gamma} f(w) \sqrt{d w}\left(\frac{\sqrt{d z} \sqrt{d w}}{w-z}-\frac{\sqrt{d \bar{z}} \sqrt{d \bar{w}}}{\bar{w}-\bar{z}}\right) .
$$

In view of the Möbius-invariance of these operators it makes sense to try to relate their properties to Möbius-invariant properties of the curve $\gamma$.

Important Möbius-invariant geometric quantities attached to $\gamma$ include the following.

Cross-ratio: The cross-ratio $\operatorname{CR}(a, b, c, d)=\frac{(c-a)(d-b)}{(d-a)(c-b)}$ of four points along $\gamma ;$ the cross-ratio satisfies the transformation law

$$
\mathrm{CR}(T a, T b, T c, T d)=\mathrm{CR}(a, b, c, d) .
$$

Kerzman-Stein "distance": For $z, w \in \gamma$ this is the quantity $\theta(z, w)$ given by the formula

$$
\begin{aligned}
\theta(z, w) & =\arg T_{\gamma} z+\arg T_{\gamma} w-2 \arg (w-z) \\
& =\operatorname{Im} \log \frac{T_{\gamma} z \cdot T_{\gamma} w}{(w-z)^{2}}
\end{aligned}
$$

where $T_{\gamma} z$ and $T_{\gamma} w$ are the forward tangent directions at $z$ and $w$, respectively.

This is a Möbius-invariant quantity admitting the following Möbiusinvariant geometric interpretation: $\theta(z, w)$ is the angle between the circle passing through $z$ and $w$ and tangent to $\gamma$ at $z$ and the circle passing through $z$ and $w$ and tangent to $\gamma$ at $w$. (See $[\mathrm{KeSt}, \S 7]$ for a more euclidean geometric interpretation.)

The definition of $\theta(z, w)$ given above doesn't make sense when $z=w$ but the approximation (6) below shows that $\theta$ extends continuously to the diagonal if we set $\theta(z, z)=0$.

At first $\theta$ seems to take values in $\mathbb{R} /(2 \pi \mathbb{Z})$. However, it is easy to check that $\theta(z, w) \equiv 0$ when $\gamma$ is a circle, and a deformation argument then shows that $\theta(z, w)$ may be uniquely defined as a continuous $\mathbb{R}$-valued function vanishing on the diagonal.

Note that $\theta$ is not a metric in general; it can vanish off the diagonal and can take negative values.

The formula

$$
\theta(z, w)=\pi+\lim _{\substack{\gamma \ni \zeta \backslash z \\ \gamma \ni \omega \backslash w}} \arg \operatorname{CR}(z, w, \zeta, \omega)
$$


shows that $\theta$ may be viewed as a partial infinitesimalization of the cross-ratio. (The notations $\zeta \searrow z$ and $\omega \searrow w$ are to indicate that $\zeta$ and $\omega$ approach $z$ and $w$ from the forward side.)

(One way to prove the geometric characterization of $\theta$ given above is to first establish Möbius invariance using (4) above or (9) below and then to reduce to the case where one of the circles is the extended real axis.)

Schwarzian derivative: The Schwarzian derivative

$$
\mathcal{S} \gamma_{*}=\frac{\gamma_{*}^{\prime \prime \prime}}{\gamma_{*}^{\prime}}-\frac{3}{2}\left(\frac{\gamma_{*}^{\prime \prime}}{\gamma_{*}^{\prime}}\right)^{2}
$$

of a parameterization $\gamma_{*}$ of an $\operatorname{arc}$ of $\gamma$ satisfies the following transformation laws:

$$
\begin{aligned}
\mathcal{S}\left(T \circ \gamma_{*}\right) & =\mathcal{S} \gamma_{*} \\
\mathcal{S}\left(\gamma_{*} \circ \phi\right) & =\left(\mathcal{S} \gamma_{*} \circ \phi\right) \cdot\left(\phi^{\prime}\right)^{2}+\mathcal{S} \phi \\
\operatorname{Im} \mathcal{S}\left(\gamma_{*} \circ \phi\right) & =\left(\operatorname{Im} \mathcal{S} \gamma_{*} \circ \phi\right) \cdot\left(\phi^{\prime}\right)^{2},
\end{aligned}
$$

where $T$ is a Möbius transformation and $\phi$ is a diffeomorphism of real intervals.

The expansions

$$
\begin{aligned}
\theta\left(\gamma_{*}(t), \gamma_{*}(u)\right)= & \frac{1}{6}(u-t)^{2} \operatorname{Im} \mathcal{S} \gamma_{*}(t)+o\left(|u-t|^{2}\right) \\
\operatorname{CR}\left(\gamma_{*}(r), \gamma_{*}(t), \gamma_{*}(u), \gamma_{*}(v)\right)= & \operatorname{CR}(r, t, u, v) \\
& +\frac{1}{6}(t-r)(v-u) \mathcal{S} \gamma_{*}(t) \\
& +o\left(|t-r|^{2}+|u-t|^{2}+|v-u|^{2}\right) \\
\operatorname{Im~CR}\left(\gamma_{*}(r), \gamma_{*}(t), \gamma_{*}(u), \gamma_{*}(v)\right)= & \frac{1}{6}(t-r)(v-u) \operatorname{Im} \mathcal{S} \gamma_{*}(t) \\
& +o\left(|t-r|^{2}+|u-t|^{2}+|v-u|^{2}\right)
\end{aligned}
$$

show that the imaginary part of the Schwarzian may be viewed as an infinitesimalization of $\theta$ or as a complete infinitesimalization of the imaginary part of the cross-ratio.

Inversive arc-length: From (5) we see that $\sqrt{\left|\operatorname{Im} \mathcal{S} \gamma_{*}(t)\right|} d t$ defines a parametrization-independent Möbius-invariant 1-form on $\gamma$. The integral

$$
\int_{\gamma} \sqrt{\left|\operatorname{Im} \mathcal{S} \gamma_{*}(t)\right|} d t
$$

is called the inversive arc-length of $\gamma$ ([Lie], [Pat], [CaSh], [Mae1]).

From (6) we see that the inversive arc-length of $\gamma$ may also be viewed as the limit of Riemann sums

$$
\sum_{j} \sqrt{6\left|\theta\left(z_{j}, z_{j+1}\right)\right|}
$$


In terms of the euclidean curvature $\kappa$ and arc-length $d s$, the inversive arclength of $\gamma$ may be written as

$$
\int_{\gamma} \sqrt{|d \kappa d s|}=\int_{\gamma} \sqrt{\left|\frac{d \kappa}{d s}\right|} d s .
$$

Inversive curvature: Consider an arc of $\gamma$ on which $d \kappa \neq 0$. If we reparameterize the arc by inversive arc-length we will have $\operatorname{Im} \delta \gamma_{*}(t)= \pm 1$. Since this parameterization is unique up to precomposition with a translation of $\mathbb{R}$, the real part of $\mathcal{S} \gamma_{*}$ becomes a scalar Möbius invariant known as the inversive curvature $\kappa_{\text {inv }}$ of $\gamma$ ([Pat], [Mae1], [Mae2]).

Writing euclidean curvature $\kappa$ as a function of euclidean arc-length as above, the inversive curvature may be written in the form

$$
\kappa_{\mathrm{inv}}=\frac{4\left(\kappa^{\prime \prime \prime}-\kappa^{2} \kappa^{\prime}\right) \kappa^{\prime}-5\left(\kappa^{\prime \prime}\right)^{2}}{8\left(\kappa^{\prime}\right)^{3}} .
$$

Our arc can be reconstructed up to post-composition with a Möbius transformation from the inversive curvature (viewed as a function of inversive arc-length) by solving the equation

$$
\mathcal{S} \gamma_{*}= \pm i+\kappa_{\mathrm{inv}}
$$

[Leh, Thm. II.1.1].

Note that the four-vertex theorem guarantees that our Jordan curves $\gamma$ will have at least four troublesome points where $d \kappa=0$ (see for example [CaSh, $\S 3])$.

The expansion (6) can be extended to read

$$
\theta\left(\gamma_{*}(t), \gamma_{*}(u)\right)= \pm \frac{1}{6}(u-t)^{2} \pm \frac{1}{180}(u-t)^{4} \kappa_{\mathrm{inv}}(t)+o\left(|u-t|^{4}\right)
$$

for an arc parameterized by inversive arc-length. Note in particular that this shows that $\sqrt{|\theta|}$ will satisfy a local triangle inequality or a local reverse triangle inequality depending on whether $\kappa_{\text {inv }}$ is negative or positive.

Returning finally to the Cauchy integrals we find that the Cauchy kernel

$$
C(z, w)=\frac{1}{2 \pi i} \frac{\sqrt{d z} \sqrt{d w}}{w-z}
$$

restricted to $\gamma \times \gamma$ satisfies

$$
2 \arg C(z, w)=\theta(z, w)+\pi .
$$

(To clarify the meaning of (9), write $z=\gamma_{*}(t), w=\gamma_{*}(u)$; then $C(z, w)=$ $\frac{1}{2 \pi i} \frac{\sqrt{\gamma_{*}^{\prime}(t)} \sqrt{\gamma_{*}^{\prime}(u)}}{w-z} \sqrt{d t} \sqrt{d u}$, and $\arg C(z, w)$ denotes the argument of the scalar function $\frac{1}{2 \pi i} \frac{\sqrt{\gamma_{*}^{\prime}(t)} \sqrt{\gamma_{*}^{\prime}(u)}}{w-z}$. To verify (9) note that $2 \arg C(z, w)=\arg C(z, w)^{2}=$ $\arg \frac{-1}{4 \pi^{2}} \frac{d z d w}{(w-z)^{2}}$.) 
The adjoint kernel

$$
\overline{C(w, z)}=\frac{1}{2 \pi i} \frac{\sqrt{d \bar{z}} \sqrt{d \bar{w}}}{\bar{w}-\bar{z}}
$$

satisfies

$$
\begin{aligned}
|\overline{C(w, z)}| & =|C(z, w)| \\
\arg \overline{C(w, z)} & =\arg C(z, w)-\theta(z, w) \\
\overline{C(w, z)} & =e^{-i \theta(z, w)} C(z, w) .
\end{aligned}
$$

For the Kerzman-Stein kernel

$$
\begin{aligned}
A(z, w) & =C(z, w)-\overline{C(w, z)} \\
& =2 i \sin \frac{\theta(z, w)}{2} e^{-i \theta(z, w) / 2} C(z, w)
\end{aligned}
$$

we have the following results.

TheOREM 1. The kernel $2 \pi \sqrt{6|A(z, w) C(z, w)|}$ extends to a continuous kernel on $\gamma \times \gamma$; the trace of the extended kernel along the diagonal is the element of inversive arc-length.

Proof. Setting $z=\gamma_{*}(t), w=\gamma_{*}(u)$ and quoting (6) we have

$$
\begin{aligned}
& 2 \pi \sqrt{6|A(z, w) C(z, w)|}=4 \pi \sqrt{3\left|\sin \frac{\theta(z, w)}{2}\right|}|C(z, w)| \\
&=\left(\sqrt{\left|\operatorname{Im}\left(\mathcal{S} \gamma_{*}\right)(t)\right|}|u-t|+o(|u-t|)\right) \\
& \cdot \frac{\sqrt{\left|\gamma_{*}^{\prime}(t)\right|\left|\gamma_{*}^{\prime}(u)\right|} \sqrt{d t} \sqrt{d u}}{\left|\gamma_{*}(t)-\gamma_{*}(u)\right|} \\
& \\
& \rightarrow \sqrt{\left|\operatorname{Im}\left(\mathcal{S} \gamma_{*}\right)(t)\right|} d t
\end{aligned}
$$

as $u \rightarrow t$.

Theorem 2. $|A(z, w)|^{2}=-\frac{1}{4 \pi^{2}} \tan \frac{\theta}{2} d_{z} d_{w} \theta$.

Proof. Differentiating (3) with respect to $z$ and $w$ and quoting (9) we have

$$
\begin{aligned}
d_{z} d_{w} \theta & =8 \pi^{2} \operatorname{Im} C(z, w)^{2} \\
& =-8 \pi^{2} \sin \theta(z, w) \cdot|C(z, w)|^{2} .
\end{aligned}
$$

Combining this with (10) we obtain the theorem.

Theorem 3. The Hilbert-Schmidt norm $\left\|\mathcal{A}_{\gamma}\right\|_{H-S}^{2}=\int_{\gamma \times \gamma}|A(z, w)|^{2}$ equals $-\frac{1}{4 \pi^{2}} \int_{\gamma \times \gamma} \tan \frac{\theta}{2} d_{z} d_{w} \theta$.

Proof. This follows from the previous result by integration. $\square$

TheOrem 4 . If the function $\theta(z, w)$ takes values in the interval $(-\pi, \pi)$ then

$$
\left\|\mathcal{A}_{\gamma}\right\|_{H-S}^{2}=\frac{1}{2 \pi^{2}} \int_{\gamma \times \gamma} d_{z} \psi d_{w} \psi
$$


where $\psi=\log \left(\sec \frac{\theta}{2}+\tan \frac{\theta}{2}\right)$.

Proof. This follows from the previous result using integration by parts and $d \psi=$ $\frac{1}{2} \sec \frac{\theta}{2} d \theta$. प

Example. Consider the "stadium domain" obtained by capping a strip of unit height and length $M$ with two semi-circles of unit diameter.

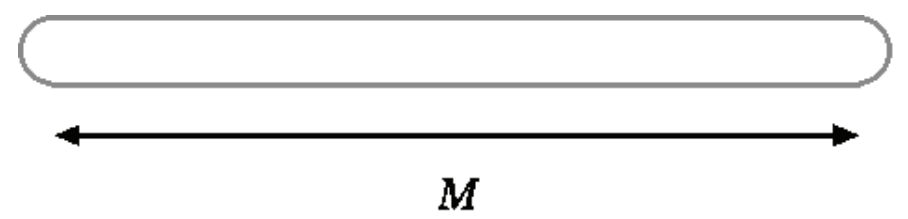

It is easy to check using Theorem 4 that $\left\|\mathcal{A}_{\gamma}\right\|_{H-S}^{2} \approx C \cdot M$ for $M$ large. The inversive arc-length of $\gamma$, however is zero!

(Actually, this is cheating a bit since $\gamma$ is piecewise $C^{3}$ but not actually $C^{3}$. However, $\gamma$ can be approximated by $C^{3}$ curves with arbitrarily small inversive arclength without any significant change to $\left.\left\|\mathcal{A}_{\gamma}\right\|_{H-S}.\right) \mathbf{u}$

The example shows that it is problematic to represent $\left\|\mathcal{A}_{\gamma}\right\|_{H-S}^{2}$ as the integral over $\gamma$ of any purely local Möbius-invariant quantity. But $\left\|\mathcal{A}_{\gamma}\right\|_{H-S}^{2}$ can be controlled by a non-local variant of inversive arc-length times a "diameter" factor based on total variation.

Theorem 5. Assume that $\theta(z, w)$ takes values in the interval $(-\pi, \pi)$ and let

$$
\begin{aligned}
L_{1} & =\max _{z \in \gamma} \int_{w \in \gamma}\left|d_{w} \psi(z, w)\right|, \\
L_{2} & =\int_{z \in \gamma} \max _{w \in \gamma}\left|d_{z} \psi(z, w)\right|=\int_{w \in \gamma} \max _{z \in \gamma}\left|d_{w} \psi(z, w)\right| .
\end{aligned}
$$

Then

$$
\left\|\mathcal{A}_{\gamma}\right\|_{H-S}^{2} \leq \frac{L_{1} L_{2}}{2 \pi^{2}}
$$

Note that $L_{1}$ is the maximum over $z \in \gamma$ of the total variations of the functions $\psi(z, \cdot)$

Proof. By (11) we have

$$
\begin{aligned}
\left\|\mathcal{A}_{\gamma}\right\|_{H-S}^{2} & =\frac{1}{2 \pi^{2}} \int_{\gamma \times \gamma} d_{z} \psi d_{w} \psi \\
& \leq \frac{1}{2 \pi^{2}} \int_{z \in \gamma} \max _{w}\left|d_{z} \psi\right| \int_{w \in \gamma}\left|d_{w} \psi\right| \\
& \leq \frac{1}{2 \pi^{2}} \int_{z \in \gamma} L_{1} \max _{w}\left|d_{z} \psi\right| \\
& =\frac{L_{1} L_{2}}{2 \pi^{2}} .
\end{aligned}
$$


Note that $L_{1} \leq L_{2}$; thus (12) implies

$$
\left\|\mathcal{A}_{\gamma}^{2}\right\|_{H-S} \leq \frac{L_{2}^{2}}{2 \pi^{2}}
$$

TheOREM 6. If $\theta(z, w)$ takes values in the interval $(-\pi, \pi)$ and the quantity $L_{2}$ given above is $<\sqrt{2} \pi$ then the Szegö projection operator for $\gamma$ is represented by the sum

$$
\sum_{j=0}^{\infty}(-1)^{j}\left(\mathrm{e}_{\gamma}+\frac{1}{2} I\right) \mathcal{A}_{\gamma}^{j}
$$

Proof. By (13) we have $\left\|\mathcal{A}_{\gamma}\right\|_{H-S}<1$. The conclusion (14) follows by quoting Theorem 8.1(a) of [KeSt].

Returning to the stadium domains discussed above we find that $L_{1} \approx C \log M$ and $L_{2} \approx C M$ for $M$ large. Thus (13) is a factor of $M$ away from being sharp for this family while (12) is only off by a factor of $\log M$.

See $[\mathrm{Bol}]$ for lower bounds for $\left\|\mathcal{A}_{\gamma}\right\|_{H-S}$.

It would be interesting to know if the quantity $L_{2}$ has any general utility in Möbius-invariant geometric analysis.

Note: The current authors are preparing a longer work which will examine the inversive arc-length in parallel with a number of other invariant arc-lengths such as the affine arc-length of Blaschke ([Bla], [Buc]).

\section{REFERENCES}

[Bla] W. Blaschke, Vorlesungen über Differentialgeometrie II: Affine Differentialgeometrie, Springer Verlag, 1923.

[Bol] M. Bolt, A lower estimate for the norm of the Kerzman-Stein operator, J. Integral Equations Appl. (to appear).

[Buc] S. Buchin, Affine Differential Geometry, Science Press, Beijing/Gordon \& Breach Science Publishers, New York, 1983.

[CaSh] G. CAIRns And R. W. Sharpe, On the inversive differential geometry of plane curves, L'Enseignement Mathématique, 36 (1990), pp. 175-196.

[Hen] P. Henrici, Applied and computational complex analysis, vol. 3, Wiley, 1986.

[KeSt] N. Kerzman And E. M. Stein, The Cauchy kernel, the Szegö kernel, and the Riemann mapping function, Math. Ann., 236 (1978), pp. 85-93.

[Leh] O. Lehto, Univalent functions and Teichmüller spaces, Springer-Verlag, 1987.

[Lie] H. Liebmann, Beiträge zur Inversionsgeometrie der Kurven, Münch. Ber. (Akademie der Wissenschaften, Munich, Sitzungsberichte), 1923, pp. 79-94.

[Mae1] J. MaedA, Differential Möbius geometry of plane curves, Jap. J. Math., 18 (1942), pp. 67260.

[Mae2] J. MAEDA, Geometrical meanings of the inversion curvature of a plane curve, Jap. J. Math., 16 (1940), pp. 177-232.

[Pat] B. PAtterson, The differential invariants of inversive geometry, Amer. J. Math., 50 (1928), pp. 553-568. 
D. E. BARRETT AND M. BOLT 